\title{
Programmable Big Data Processing Framework to Reduce On-Chip Communications and Computations Towards Reducing Energy of the Processing
}

\author{
Anusha Medavaka ${ }^{1}$ \\ Software Programmer, Seven Hills IT Solutions LLC, $\mathrm{NJ}^{1}$
}

\begin{abstract}
Multi-Sensor health and wellness tracking systems are utilized to forecast future occasions of our health and wellness system. Each sensor produces a whopping quantity of data per 2 nd and also requires to be refined in real-time. At the very same time, health and wellness tracking systems are battery ran, hence they have stiff restraints on power and also the location of the processing platform. Furthermore, health and wellness surveillance systems need to be precise, hence we adjust artificial intelligence methods to boost discovery precision. We recommend a programmable Big Data Processing structure to decrease on-chip interactions as well as calculations, therefore minimizing the power of the processing. We incorporate a low-overhead laying out structure with a low-power programmable PENC many-core platform. The laying out method decreases the data interactions and also calculations, furthermore processing time is reduced by parallel processing on the many-core platform. For the presentation we reveal seizure discovery application with 22-channel of electroencephalograph (EEG), each network creates 256 examples per 2nd calling for overall of 88 Kbps data price. The calculations are lowered by 16 while the power intake of processing is minimized approximately $68 \%$. For compression prices of $2-16$, the seizure discovery efficiency for the level of sensitivity and also uniqueness is broken down by $2.07 \%$ and also $2.97 \%$, specifically for Logistic Regression classifier.
\end{abstract}

Keywords: Sketching Technique, Big Data Processing, Seizure Detection

\section{INTRODUCTION}

Real-time applications such as health and wellness surveillance and also video clip security create an enormous quantity of data every hr at an unmatched price. The created big data collections are made use of for decreasing danger, recognizing unidentified things and also discover concealed patterns. To manipulate concealed data patterns and also accomplish effective forecasts, Artificial intelligence (ML) strategies are taken on. As an example, in clever as well as wearable wellness surveillance tools, ML methods are embraced to forecast future carcinogen such as boosted high blood pressure, blood-glucose degrees, drop discovery as well as seizure discovery [1], [2], [3] To offer reliable wellness keeping track of a multi-sensor strategy is embraced, where each sensor generates multitude of examples per secondly. At every example duration, keeping track of tool requires to refine a number of DSP and also ML bits. Therefore strict real-time restraints require rapid processing platform whereas wearable battery ran gadget demands an incredibly power reliable platform. Individualized biomedical tools normally trick- consists of 3 major circuit blocks: 1 . Signal Purchase obstruct consisting of analog to the electronic converter, 2. Digital Signal Refine- ing block which generally has Function extractor and also ML Classifiers 3. Radio transmitter to transfer the refined data or forecast to the individual or clinical employees. In this paper, we suggest a sketching-based big data processing structure that carries out all signal processing in your area at the sensor. We incorporate a low-overhead mapping out structure with domain name details programmable PENC many-core accelerator to reduce calculation power [4] Whereas Mapping out strategy at the front end lowers variety of data transfers, calculations, and also storage. The laying out algorithm decreases data by getting a linear mix of the data prior to processing. Data decrease by taking on Sketching-based structure triggers 2 essential obstacles that require to be dealt with 1 . ideal category mistake 2 . reduced equipment expenses for Map out the execution. To show the effectiveness of the structure, we use the suggested structure for multi-channel seizure discovery application. A multi-channel seizure discovery tracking system creates a massive quantity of EEG data, which requires to be refined on-chip in real-time with reduced power usage.

Feature extraction and also distinction is actually carried out regionally at the sensor [1] Hence, it transfers a single little bit prophecy i.e confiscation or even no-seizure, this lowers gearbox energy dramatically. Furthermore, the transmitter may be in the sleeping method while processing. Figure 1 reveals designed Sketching-based big data processing platform. To Begin With, Deterministic Sketching strategy is actually embraced for data decrease. At that point, the thin data 


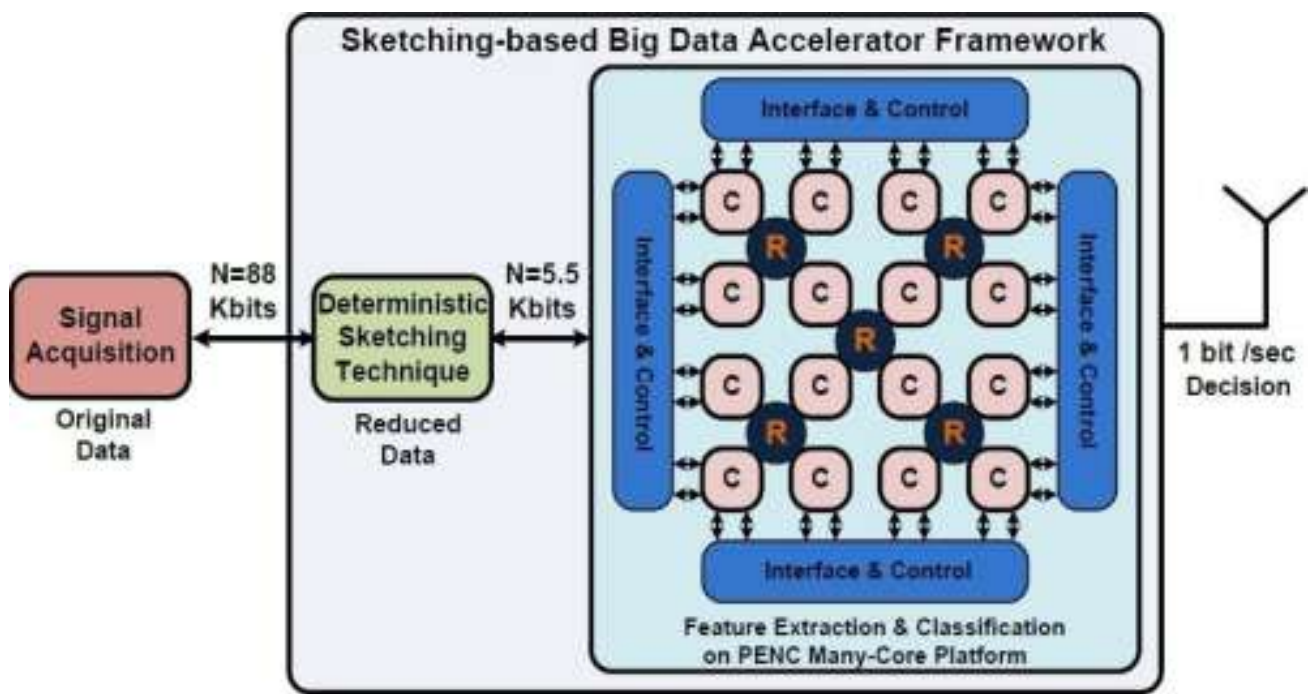

Fig. 1. Proposed Sketching-based Big Data Acceleration Framework, where C-Cluster of cores, R- Hierarchical router

collection is actually nourished to the feature extraction as well as category component implemented on programmable PENC many-core platform. The laying out- located structure lessens a lot of input examples for feature extraction thereby minimizing processing opportunity. Lastly, the prophecy is actually sent to the individual or even health care staffs. Taking over AFE takes 256 examples 16 little bits each, the transmitter fee may possibly transform coming from 88 Kbits/s (for 22-channels).

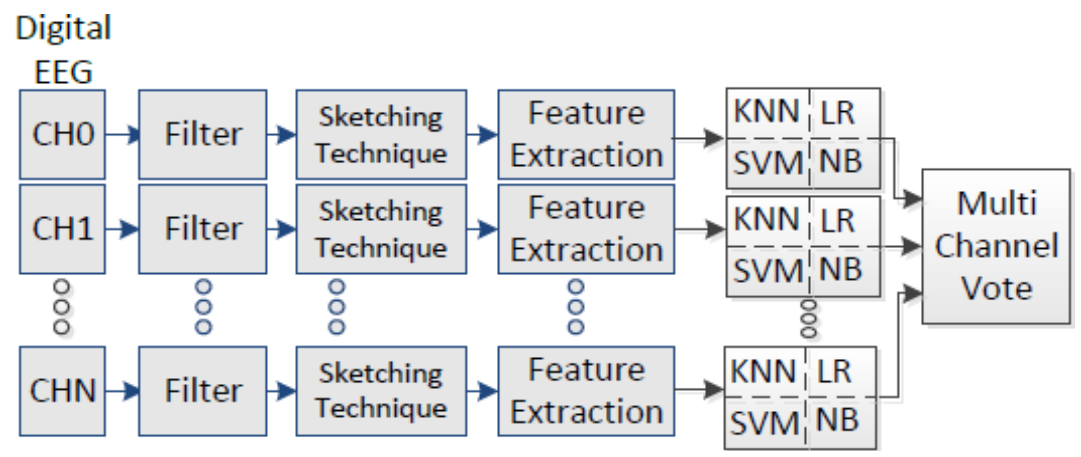

Fig. 2. Block diagram of the multi-channel seizure detection processor containing Sketching kernel (to reduce data rate), feature extraction, ML classifier, and multi-channel vote to $1 \mathrm{bit} / \mathrm{s}$

\section{SKETCHING-BASED FRAMEWORK FOR BIG DATA PROCESSING}

In this particular segment, our experts for a while review sketching algorithm, feature extraction, ML protocols category and also confiscation diagnosis method.

A. Sketching Algorithm: In Outlining, the data is actually gotten at a cost symmetrical to info cost, i.e it acquires straight mixtures of the data instead of lessening the variety of examples [5] The essential concept responsible for Outlining depend on resolving formula 1 . Allow $\varphi$ be actually the size source of measurement $\mathrm{M}$, where $\mathrm{M}$ is actually the amount of dimensions to become taken as well as $\mathrm{N}$ is actually the size of the indicator as well as $\mathrm{x}$ be actually a m-sparse sign of duration $\mathrm{N}$. Increasing these pair of angles turnouts $\mathrm{y}$ of $\operatorname{span} \mathrm{M}$, which has the sizes acquired $\mathrm{b} \times \mathrm{y}$ the estimate of $\mathrm{x}$ right into $\varphi$.

$$
\mathrm{Y}=\varphi \mathrm{X}
$$

Typically Dimension source $\varphi$ is actually created utilizing Bernoulli or even iid Gaussian procedure which delight Restricted Isometric Quality along with higher likelihood [6] Having said that, creating Bernoulli or even iid Gaussian sources in equipment is actually facility. Consequently, our company takes on the deterministic arbitrary source to lessen components cost in regards to the region and also power needs. Deterministic arbitrary source is actually created through arbitrarily deciding on a part of the rows of an identification source [7] Figure 4D reveals a reconfigurable laying out architecture utilizing a DRM procedure for various input indicator measurements. A block RAM is actually utilized to hold one home window of input sign for testing reason. Undoubtedly, in this particular strategy, there is actually no demand to spare all nos as well as ones in the ROM. In previous job [1], the writers that the mapping out construction 
utilizing DRM strategy reduces the equipment cost matched up to outlining element making use of LFSR circuit [8], [9]. $\rightarrow$ SVM $\rightarrow$ Naive Bayes-*.Logistic Regression $-\mathrm{K}-\mathrm{NN}(\mathrm{K}=3)$
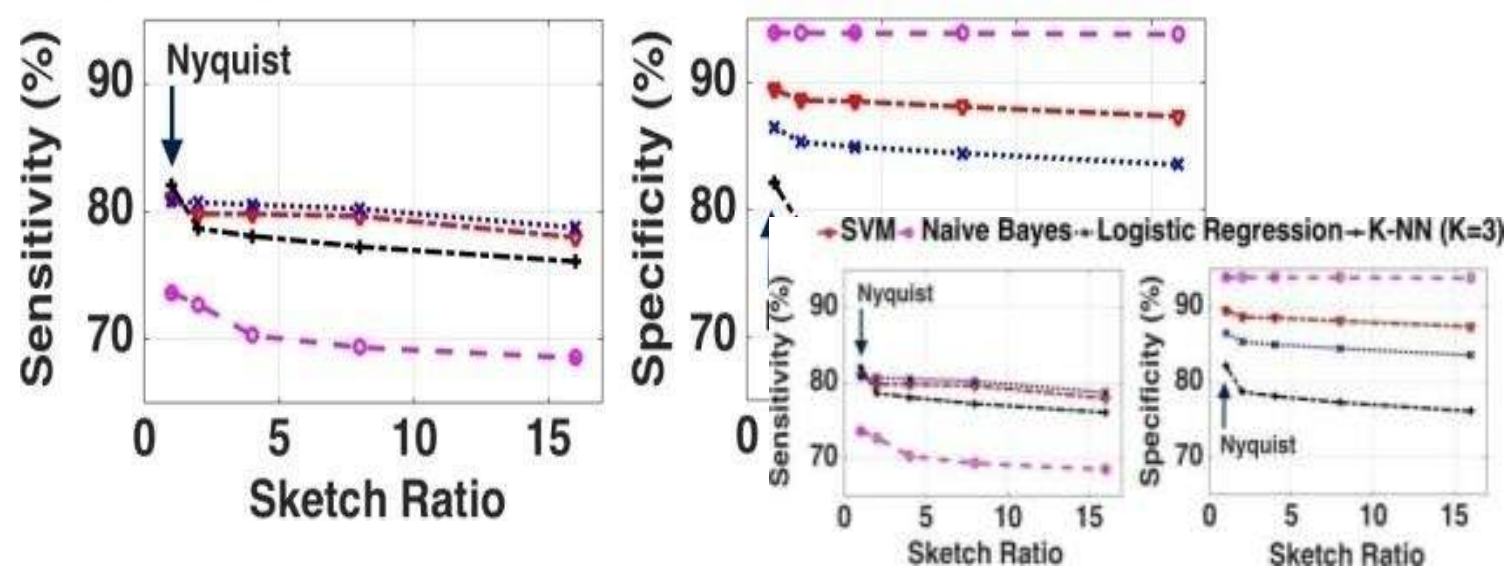

Fig. 3. Performance of the proposed seizure detection algorithm with direct use of sketched signals and employing a deterministic random matrix. Degradation in sensitivity and specificity is approximately $2.07 \%$ and $2.97 \%$, respectively, up to a sketch rate of 16 .

\section{B. Seizure Detection Approach and Performance Analysis}

The data made use of to assess the efficiency of the suggested unit contains EEG audios are actually picked up at the Children's Healthcare facility Boston ma [10] Figure 2 presents the block representation for the Confiscation Discovery along with a combination of sketching-based structure. The data acquired coming from EEG scalp remains in the kind of uncooked opportunity set, using uncooked data to classifiers will certainly lead to reduced precision. Hence the EEG sensor data is actually gone through a filter to clear away higher regularity and also DC elements. This data is actually made use of as the input to the suggested Sketching algorithm for various Layout Proportion, as much as 16x. A home window of 256 examples (one 2nd every network) is actually selected for the input of Sketching Algorithm. At that point, the sketched data are actually utilized to produce 5 easy attributes for each and every next of EEG data every station [2] Within this research study our team take into consideration region under contour, stabilized degeneration, line span, typical peak bigness, as well as common lowland bigness as attributes [2], [11] The strategies for these 5 components are actually given up Desk

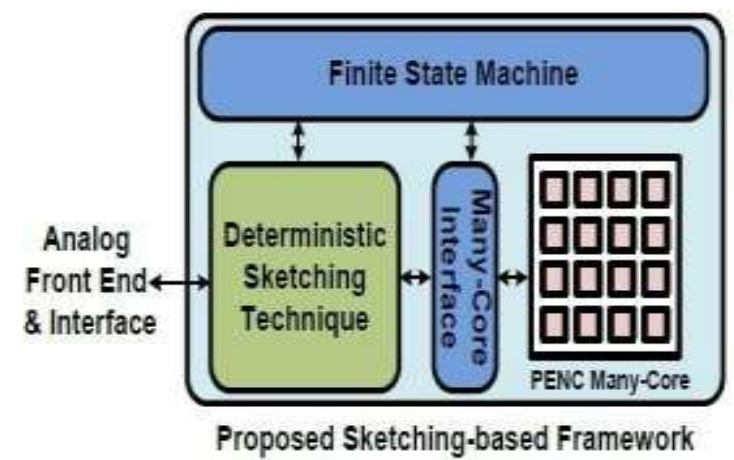

(A)

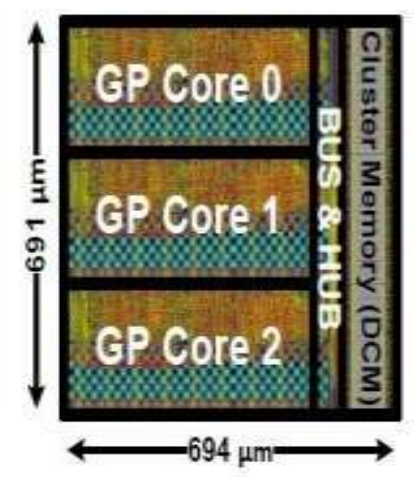

(B)

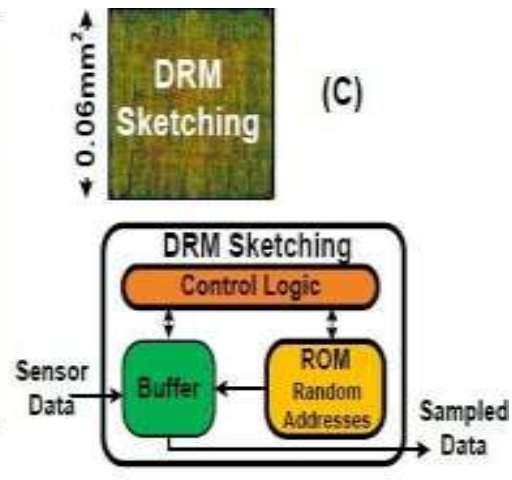

(D)

Fig. 4. (A) Block Diagram of The Proposed Big Data Accelerator for Seizure Detection Application, where Many-Core Platform performs Feature Extraction, Classification and Multi-Channel Vote. Note:

SPI and AFE blocks are not implemented (B) Layout of Bus-based Cluster of PENC many-core platform in $65 \mathrm{~nm}$ CMOS technology, where GP is General Purpose core (C) Layout of Sketching Module in 65 nm CMOS technology

(D) Block Diagram of Deterministic Random Matrix based Sketching technique

The suggested DRM outlining strategy decreases feature extraction calculations which subsequently minimizes general confiscation diagnosis energy and time intake. Each stations' functions are actually at that point identified utilizing some of 4 classifiers: K-Nearest Neighbors (KNN), Help Angle Machines, Gullible Bayes, or even Logistic Regression (LR). A last is actually at that point made use of to generate a decision based upon a multichannel ballot plan. The efficiency of the confiscation sensor is actually defined in regards to sensitiveness and also uniqueness. Level of sensitivity pertains to the percent of confiscation beginnings recognized and also Uniqueness pertains to the portion of improperly sensed 
confiscation starts [1]. Likewise, to predict the sensor efficiency on the data coming from an individual, a leave-one record-out cross-validation method is actually made use of [12] The suggested device could possibly attain a sensitiveness of $81.8 \%$ as well as the uniqueness of $93.9 \%$ for Nyquist-domain confiscation data (without Outlining). Figure 3 presents the confiscation sensor functionality utilizing sketched data for SR coming from 2 approximately 16 As it may be viewed coming from the Figure 3, the deterioration in sensitiveness and also uniqueness is actually roughly $2.07 \%$ as well as $2.97 \%$, specifically, around a laying out fee of 16 .

\section{BIG DATA “ACCELERATION" MANY-CORE PLATFORM}

A. PENC: Many-Core Architecture: The suggested PENC many-core style includes in- order CPUs along with a 6 phase pipe, a RISC like DSP directive collection as well as a Harvard mind style. The primary operates a 16-bit data pathway along with a very little direction as well as data memory ideal for duty degree similarity. Moreover, the center possesses a reduced complexity, lessened direction readied to even lower location and also energy impact. These processing primaries can easily carry out the calculation, division, and also inter-core interaction guidelines. In this particular style, every 3 primaries along with a moment are actually arranged right into a cluster which can easily execute intra-communication straight with a bus and also inter-communication via a transmitting style. The many-core is actually entirely executed in Verilog and also put and also directed in 65nm, 1V CMOS Technology [4] Figure 4B reveals the style of a bus-based cluster of PENC several- primary platform. Our many-core progression setting features an architecture simulation filled in Espresso. The simulation functions as a recommendation execution of the style; its own function is actually to produce screening, refining, as well as boosting the design less complicated. It designs the functions of the processor chip and also works out the ultimate condition of sign up and also data moments. It discloses studies including the variety of patterns demanded ALU, division, and also interaction guidelines. The implementation opportunity evaluation is actually computed by utilizing many-core simulation, whereas for energy evaluation, the protocols are actually carried out on the version of the component of the many-core platform as well as a substitute utilizing Rhythmus NC- Verilog. The task element is actually after that obtained and also is actually made use of due to the Tempo Conflict device for precise energy calculation. The PENC many-core design is actually preferably matched for many biomedical requests as it attends to the features as well as difficulties fundamental along with these uses [4], [Thirteen] [14].

B. ML Algorithm Implementations on Many-Core Platform: The seizure discovery application consists of both feature extraction as well as category. A solitary core does feature extraction on home windows of examples from 22 networks in serial. It creates an examination vector of 5 attributes for each and every home window and also relays the outcomes to the cores that do category. We checked out 4 various monitored ML formulas relative to implementation time, power usage and also its storage memory demands. For the SVM classifier, assistance vectors are dispersed equally amongst 47 cores in 16 collections. Each core calculates the dot item of the examination vector with 106 assistance vectors and also collects the amount of the outcomes. After each core has actually finished its section of the job, the cores connect to calculate the complete amount and also one core establishes the last category. The PENC should perform at $160 \mathrm{kHz}$ to procedure 22 home windows of 256 examples in one 2nd, and also at $157 \mathrm{kHz}$ in the instance of 32 examples per home window. KNN is mapped on 47 cores and also the PENC should perform at $228 \mathrm{kHz}$ without laying out and also at $224 \mathrm{kHz}$ in instance SR $=16 . \mathrm{NB}$, as well as LR classifiers, learn offline, therefore just weight vectors are kept on the cache memory. The seizure discovery is after that carried out by taking dot item of brand-new seizure data and also weight vector.

\section{IMPLEMENTATION RESULTS}

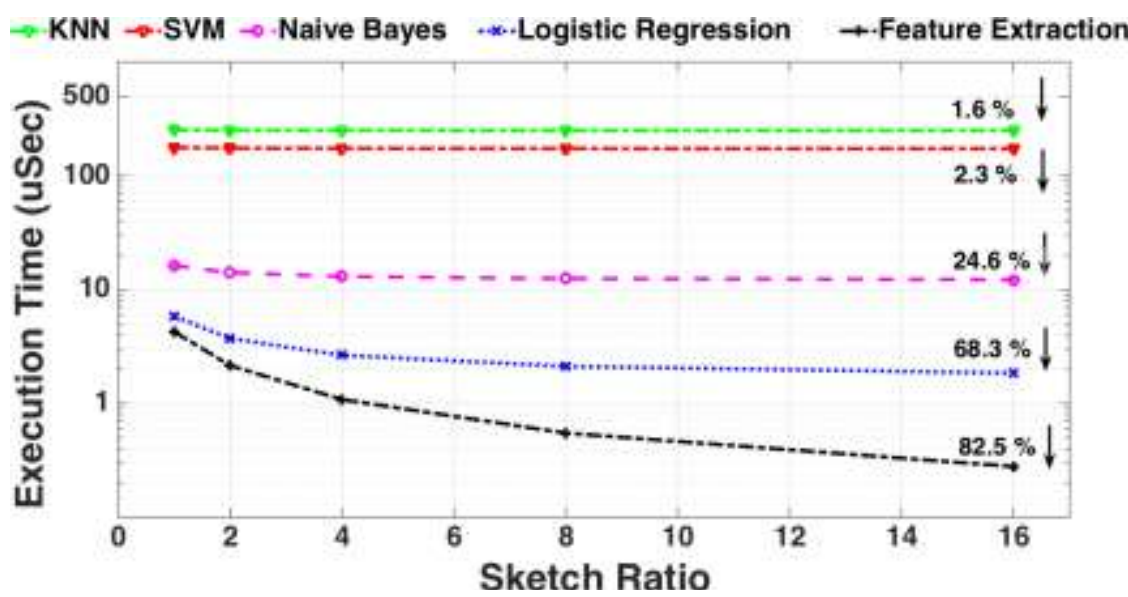

Fig. 5. Execution time analysis for the proposed system using different ML classifiers on the many-core platform 


\title{
International Journal of Advanced Research in Computer and Communication Engineering
}

\author{
Vol. 8, Issue 4, April 2019
}

The outcomes reveal that Sketching-based structure decreases the computational complexity of Feature extraction bit reducing computational latency as much as $82 \%$ for $\mathrm{SR}=16$ as received Figure 5. It likewise reveals implementation time evaluation for each and every ML classifier with feature extraction, hence total seizure discovery time is lowered as much as $68 \%$. Table I reveals the power evaluation of ML algorithm mapping with feature extraction on the PENC many-core platform. KNN, as well as SVM formulas, call for a lot of cores as well as they take 10,181 and also 7,056 cycles specifically to implement, therefore it conceals the benefit of computational complexity decrease for feature extraction bit. Nevertheless, NB as well as LR reveal $24 \%$ to $68 \%$ renovation in power and also implementation time.

Table I: Energy Analysis of PENC Many-Core ML Algorithm Mapping with the Proposed Big Data Acceleration Framework

\begin{tabular}{|c|c|c|c|c|c|}
\hline \multirow[t]{2}{*}{ Architecture } & \multirow[t]{2}{*}{$\mathrm{FE}(\mathrm{mJ})$} & \multicolumn{4}{|c|}{ FE + ML Classification (mJ) } \\
\hline & & KNN & SVM & NB & LR \\
\hline W/o Sketching & 0.032 & 1.720 & 1.202 & 0.015 & 0.0057 \\
\hline With $S R=2$ & 0.018 & 1.706 & 1.188 & 0.014 & 0.004 \\
\hline With $S R=4$ & 0.011 & 1.699 & 1.181 & 0.013 & 0.0027 \\
\hline With $\mathrm{SR}=8$ & 0.007 & 1.695 & 1.177 & 0.0122 & 0.0021 \\
\hline With $\mathrm{SR}=16$ & 0.005 & 1.693 & 1.175 & 0.0119 & 0.0018 \\
\hline
\end{tabular}

Table II: Energy consumption Comparison of FPGA with PENC Many-Core Platform for the Seizure Detection using Sketching technique, Feature Extraction and ML algorithms

\begin{tabular}{|c|c|c|c|c|c|}
\hline Architecture & SR & KNN $(\mathrm{mJ})$ & SVM $(\mathrm{mJ})$ & $\mathrm{NB}(\mathrm{mJ})$ & $\mathrm{LR}(\mathrm{mJ})$ \\
\hline PENC Many-Core & \multirow{2}{*}{1} & 1.720 & 1.202 & 0.015 & 0.0057 \\
\cline { 4 - 6 } & & 1349 & 164.64 & 0.76 & 0.64 \\
\hline Artix-7 FPGA & \multirow{2}{*}{16} & 1.693 & 1.175 & 0.0119 & 0.0018 \\
\cline { 4 - 6 } PENC Many-Core & & 1348 & 164.29 & 0.40 & 0.29 \\
\hline Artix-7 FPGA & & &
\end{tabular}

To reveal the performance of PENC many-core platform as a Big Data accelerator, we additionally executed the recommended Sketching-based structure on reduced power Xilinx Artix-7 FPGA platform. Excellent treatment was required to make certain uniformity of the formulas throughout both systems. For the Artix-7 FPGA application, the power and also tim- ing outcomes were acquired making use of Xilinx XPower as well as Timing analyzer, specifically. Table II reveals the power contrast of many-core as well as FPGA systems with and also without Mapping out strategy. For LR and also NB formulas PENC application eats around 100 much less power as contrasted to FPGA execution.

\section{CONCLUSION}

The paper suggests Big Data Processing Accelerator for Biomedical applications. The paper discovered the benefits of Sketching algorithm to minimize the computational complexity, hence decreasing implementation energy and a time Furthermore domain name details many-core platform is discovered for parallel processing of typically utilized ML formulas. The laying out- based structure minimizes the power of the seizure discovery CPU as much as $68 \%$ with minimal impact on uniqueness as well as the level of sensitivity. The recommended platform with reduced power cores and also GIRLS style enables the platform not just to manipulate the job degree as well as data-level similarity however likewise do vibrant voltage and also regularity scaling to substantially minimize power usage. The ML formulas are likewise carried out on reduced power Xilinx Artix-7 FPGA. Contrasted to FPGA application the PENC many-core, usually, eats around $100 \times$ much less power.

\section{REFERENCES}

[1]. A. Jafari et al., "A low power seizure detection processor based on direct use of compressively-sensed data and employing a deterministic random matrix," IEEE Biomedical Circuits and Systems (Biocas) Conference, 2015.

[2]. A. Page et al., "A flexible multichannel eeg feature extractor and classifier for seizure detection,” Circuits and Systems II: Express Briefs, IEEE Transactions on, vol. 62, no. 2, pp. 109-113, 2015.

[3]. A. Page, A. Kulkarni, and T. Mohsenin, "Utilizing deep neural nets for an embedded ecg-based biometric authentication system," in IEEE Biomedical Circuits and Systems (Biocas) Conference, Oct 2015.

[4]. A. Kulkarni, T. Abtahi, E. Smith, and T. Mohsenin, "Low energy sketching engines on many-core platform for big data acceleration," in Proceedings of the 26th Edition of the Great Lakes Symposium on VLSI, ser. GLSVLSI '16. New York, NY, USA: ACM, 2016.

[5]. D. L. Donoho, "Compressed sensing," Information Theory, IEEE Trans- actions on, vol. 52, no. 4, pp. 1289-1306, 2006.

[6]. A. Septimus and R. Steinberg, "Compressive sensing hardware recon- struction," Circuits and Systems (ISCAS), Proceedings of 2010 IEEE International Symposium on, pp. 3316-3319, 2010.

[7]. Anusha Medavaka, P. Shireesha, "Analysis and Usage of Spam Detection Method in Mail Filtering System" in "International Journal of Information Technology and Management”, Vol. 12, Issue No. 1, February-2017 [ISSN : 2249-4510] 


\title{
International Journal of Advanced Research in Computer and Communication Engineering
}

\author{
Vol. 8, Issue 4, April 2019
}

[8]. Anusha Medavaka, P. Shireesha, "Review on Secure Routing Protocols in MANETs" in "International Journal of Information Technology and Management”, Vol. VIII, Issue No. XII, May-2015 [ISSN : 2249-4510]

[9]. Anusha Medavaka, P.Shireesha, "Classification Techniques for Improving Efficiency \& Effectiveness of Hierarchical Clustering for the Given Data Set" in "International Journal of Information Technology \& Management", Vol.X, Issue No.XV, May-2016 [ISSN: 2249-4510]

[10]. Anusha Medavaka, P. Shireesha, "Optimal framework to Wireless Rechargeable Sensor Network based Joint Spatial of the Mobile Node" in "Journal of Advances in Science and Technology", Vol. XI, Issue No. XXII, May-2016 [ISSN : 2230-9659]

[11]. Anusha Medavaka,"Enhanced Classification Framework on Social Networks" in "Journal of Advances in Science and Technology", Vol. IX, Issue No. XIX, May-2015 [ISSN : 2230-9659]

[12]. Anusha Medavaka, P. Shireesha, "A Survey on TraffiCop Android Application" in "Journal of Advances in Science and Technology", Vol. 14, Issue No. 2, September-2017 [ISSN : 2230-9659]

[13]. Anusha Medavaka, Dr. P. Niranjan, P. Shireesha, "USER SPECIFIC SEARCH HISTORIES AND ORGANIZING PROBLEMS" in "International Journal of Advanced Computer Technology (IJACT)", Vol. 3, Issue No. 6 [ISSN : 2319-7900]

[14]. Anusha Medavaka, "Monitoring and Controlling Local Area Network Using Android APP" in "International Journal of Research", Vol. 7, Issue No. IV, April-2018 [ISSN : 2236-6124]

[15]. Yeshwanth Rao Bhandayker, "AN OVERVIEW OF THEINTEGRATION OF ALL DATA MINING AT CLOUD-COMPUTING" in "Airo International Research Journal", Volume XVI, June 2018 [ISSN : 2320-3714]

[16]. Siripuri Kiran, 'Decision Tree Analysis Tool with the Design Approach of Probability Density Function towards Uncertain Data Classification', International Journal of Scientific Research in Science and Technology(IJSRST), Print ISSN : 2395-6011, Online ISSN : 2395-602X,Volume 4 Issue 2, pp.829-831, January-February 2018. URL : http://ijsrst.com/IJSRST1841198

[17]. Yeshwanth Rao Bhandayker, "Artificial Intelligence and Big Data for Computer Cyber Security Systems" in "Journal of Advances in Science and Technology", Vol. 12, Issue No. 24, November-2016 [ISSN : 2230-9659]

[18]. Sugandhi Maheshwaram, "A Comprehensive Review on the Implementation of Big Data Solutions" in "International Journal of Information Technology and Management", Vol. XI, Issue No. XVII, November-2016 [ISSN : 2249-4510]

[19]. "Method for activating research and institutional assignments Big Data Services," National Information Society Agency, (2013).

[20]. Si-Jung Kim, "Double Secure Layers Architecture for Privacy Protection in Big Data," In proceedings of the 2014 International Conference on Platform and Service (PlatCon-14), (2014) January.

[21]. "An Analysis of Technology Demand for Big Data based Privacy Information Protection", Industry Academic Cooperation Foundation Sungshin women's university, (2012) December.

[22]. Suresh Kumar Mandala, Neelima Gurrapu, Mahipal Reddy Pulyala, “ A Study on the Development of Machine Learing in Health Analysis", Indian Journal of Public Health Research \& Development, volume 9, Number 12, December 2018, [ISSN-0976-0245(Print)-ISSN-0976-5506 (Electronic)]

[23]. Suresh Kumar Mandala,Mahipal Reddy Pulyala and Sanjay Pachouri, "Being a Smart Sapien with Information Centric Networking and Cloud Computing", International Journal of Pure and Applied Mathematics,Volume 117, No. 21, 2017, 243-255,[ISSN: 1311-8080 (printed version)]

[24]. Suresh Kumar Mandala, Sanjay Pachouri, "performance evaluation of multi stage attacks prediction", Journal of Advanced Research in Dynamical and Control Systems, Vol. 9, September 2017, JARDCS Special Issue On Engineering Technology.

[25]. Thota Mounika, Mandala Suresh kumar, "Document Proximity: Keyword Query Suggestion Based On User Location", International Journal of Research, Volume 04, Issue 14, November 2017, [e-ISSN: 2348-6848,p-ISSN: 2348-795X] .

[26]. Syeda Sobia Farees, M. Suresh Kumar, "A Novel Approach for Protecting Location Information in Geosocial Applications “, IJIEMR, Vol 1, Issue 2, November 2016 [ISSN:2456-5083]

[27]. Suresh Kumar Mandala,Sanjay Pachouri, "A Reviewed Study on Financial Cyber Crime and Frauds", International Journal of Advances in Arts, Sciences and Engineering(ijoaase.com), Volume 4 Issue 9, Sep 2016, [ISSN. 2320-6144 (Online)]

[28]. Ajmera Rajesh, Siripuri Kiran, " Anomaly Detection Using Data Mining Techniques in Social Networking" in "International Journal for Research in Applied Science and Engineering Technology", Volume-6, Issue-II, February 2018, 1268-1272 [ ISSN : 2321-9653], www.ijraset.com

[29]. Sugandhi Maheshwaram, "An Overview of Open Research Issues in Big Data Analytics" in "Journal of Advances in Science and Technology", Vol. 14, Issue No. 2, September-2017 [ISSN : 2230-9659]

[30]. Siripuri Kiran, Ajmera Rajesh, "A Study on Mining Top Utility Itemsets In A Single Phase" in "International Journal for Science and Advance Research in Technology (IJSART)", Volume-4, Issue-2, February-2018, 637-642, [ ISSN(ONLINE): 2395-1052]

[31]. Yeshwanth Rao Bhandayker, "Security Mechanisms for Providing Security to the Network" in "International Journal of Information Technology and Management", Vol. 12, Issue No. 1, February-2017, [ISSN : 2249-4510]

[32]. Sugandhi Maheshwaram, S. Shoban Babu , "An Overview towards the Techniques of Data Mining" in "RESEARCH REVIEW International Journal of Multidisciplinary", Volume-04, Issue-02, February-2019 [ISSN : 2455-3085]

[33]. Yeshwanth Rao Bhandayker, "A Study on the Research Challenges and Trends of Cloud Computing" in "RESEARCH REVIEW International Journal of Multidisciplinary ", Volume-04, Issue-02, February-2019 [ISSN : 2455-3085]

[34]. Sriramoju Ajay Babu, Dr. S. Shoban Babu, "Improving Quality of Content Based Image Retrieval with Graph Based Ranking" in "International Journal of Research and Applications", Volume 1, Issue 1, Jan-Mar 2014 [ ISSN : 2349-0020 ]

[35]. Dr. Shoban Babu Sriramoju, Ramesh Gadde, “A Ranking Model Framework for Multiple Vertical Search Domains” in "International Journal of Research and Applications" Vol 1, Issue 1,Jan-Mar 2014 [ ISSN : 2349-0020 ].

[36]. Mounika Reddy, Avula Deepak, Ekkati Kalyani Dharavath, Kranthi Gande, Shoban Sriramoju, "Risk-Aware Response Answer for Mitigating Painter Routing Attacks" in "International Journal of Information Technology and Management", Volume VI, Issue I, Feb 2014 [ ISSN : 2249-4510]

[37]. Sugandhi Maheshwaram, "A Review on Deep Convolutional Neural Network and its Applications" in "International Journal of Advanced Research in Computer and Communication Engineering", Vol. 8, Issue No. 2, February-2019 [ISSN : 2278-1021], DOI 10.17148/IJARCCE.2019.8230

[38]. Yeshwanth Rao Bhandayker. "An Overview : Security Solutions for Cloud Environment." International Journal for Scientific Research and Development 7.2 (2019): 1596-1598.

[39]. Yeshwanth Rao Bhandayker. "An Overiew Of Cyber Security", International Journal of Research, vol. 8, Issue. 3 (2019): $2236-6124$.

[40]. Sugandhi Maheshwaram, "A Study On The Challenges In Handling Big Data", International Journal of Research, vol. 8, Issue. 3 (2019): 2236-6124

[41]. Malik S., Huet F., and Caromel D., "Cooperative Cloud Computing in Research and Academic Environment using Virtual Cloud, Emerging Technologies (ICET)," in Proceedings of the International Conference on Digital Object Identifier, 2012.

[42]. Patel A., Birla M., and Nair U., "Addressing Big Data Problem using Hadoop and Map Reduce, Engineering (NUiCONE)," in Proceedings of the International Conference on Digital Object Identifier, pp. 1-5, 2012. 